\title{
The anconeus epitrochlearis muscle may protect against the development of cubital tunnel syndrome: a preliminary study
}

\author{
Thomas J. Wilson, MD, ${ }^{1}$ R. Shane Tubbs, PhD, ${ }^{2}$ and Lynda J. S. Yang, MD, PhD' \\ 1Department of Neurosurgery, University of Michigan, Ann Arbor, Michigan; and 2Seattle Science Foundation, Seattle, \\ Washington
}

OBJECTIVE The authors hypothesized that when the anatomical variant of an anconeus epitrochlearis is present, the risk of developing cubital tunnel syndrome would be reduced by replacing the normal roof of the cubital tunnel (Osborne's ligament) with a more forgiving muscular structure, the anconeus epitrochlearis. The authors further hypothesized that when the presence of an anconeus epitrochlearis contributes to ulnar neuropathy, it would be secondary to muscular hypertrophy, thereby making it more likely to occur in the dominant arm. Therefore, the goal of the present study was to evaluate these hypotheses.

METHODS This retrospective cohort study was performed by reviewing the records of all adult patients who underwent operative intervention for cubital tunnel syndrome between 2005 and 2014 as the experimental group and all asymptomatic patients in the medical literature who were part of a series reporting the prevalence of an anconeus epitrochlearis as the control group. The primary outcome of interest was the presence of an anconeus epitrochlearis in asymptomatic individuals versus patients with cubital tunnel syndrome.

RESULTS During the study period, 168 patients underwent decompression of the ulnar nerve for cubital tunnel syndrome, and an anconeus epitrochlearis was found at surgery in 9 (5.4\%) patients. The control group consisted of 634 asymptomatic patients from the medical literature, and an anconeus epitrochlearis was present in $98(15.5 \%)$ of these patients. An anconeus epitrochlearis was present significantly less frequently in the symptomatic patients than in asymptomatic individuals $(p<0.001)$. Among patients undergoing surgical decompression, an anconeus epitrochlearis was associated with symptoms in the dominant arm $(p=0.037)$.

CONCLUSIONS The authors found that an anconeus epitrochlearis was present significantly less often in patients with cubital tunnel syndrome than in asymptomatic controls. The mechanism of protection may be that this muscle decreases the rigidity of the entrance into the cubital tunnel. When an anconeus epitrochlearis does contribute to cubital tunnel syndrome, it is significantly more likely to occur in the dominant arm, possibly due to repetitive use and hypertrophy of the anconeus epitrochlearis. The presence of an anconeus epitrochlearis may be protective against the development of cubital tunnel syndrome, although this is a preliminary finding.

http://thejns.org/doi/abs/10.3171/2015.10.JNS151668

KEY WORDS anconeus epitrochlearis; cubital tunnel syndrome; ulnar nerve; peripheral nerve

$\mathrm{E}$ NTRAPMENT neuropathies commonly occur as nerves pass beneath rigid ligamentous structures. Ulnar neuropathy at the cubital tunnel is the second most common entrapment neuropathy, following carpal tunnel syndrome. In the United States, there are approximately 75,000 new cases of cubital tunnel syndrome reported annually. ${ }^{15,17}$ A number of factors that predispose the development of cubital tunnel syndrome have been suggested, including diabetes mellitus, extreme valgus or varus deformity of the elbow, repetitive tasks that require pro- tracted elbow flexion, and the repetitive use of vibrating tools ${ }^{4,6,11}$ For the ulnar nerve, the most common site of compression is at the entrance to the cubital tunnel as the nerve passes beneath Osborne's ligament, spanning from the medial epicondyle to the olecranon process of the ulna.

One anatomical variant that has been reported to be a site of compression and cause of cubital tunnel syndrome is the presence of an anconeus epitrochlearis muscle (epitrochleoanconeus, epitrochleo-olecranonis, accessory anconeus, anconeus sextus, ulnaris internis, cubital anterieur). 
When present, this muscle replaces Osborne's ligament and spans from the medial epicondyle of the humerus to the olecranon process of the ulna and serves as the roof of the cubital tunnel (Fig. 1). Several case reports and case series have reported cubital tunnel syndrome secondary to an anconeus epitrochlearis. ${ }^{8,9,13,15,19}$ It is not an infrequent variant, and if it is truly a risk factor for ulnar neuropathy, then one would expect it to be frequently reported. On the contrary, however, reports of ulnar neuropathy secondary to the presence of an anconeus epitrochlearis are limited to case reports and small case series. We hypothesized that by replacing Osborne's ligament with a more forgiving muscular structure, the anconeus epitrochlearis, the risk of developing cubital tunnel syndrome may actually be reduced. We further hypothesized that when the presence of an anconeus epitrochlearis contributes to ulnar neuropathy, it would be secondary to muscular hypertrophy, making it more likely to occur in the dominant arm. Therefore, the present study was performed to evaluate these hypotheses.

\section{Methods}

\section{Study Design}

This retrospective cohort study was approved by the institutional review board at the University of Michigan. Adult patients who underwent an initial operative inter- vention for ulnar neuropathy at the cubital tunnel between January 2005 and December 2014 were maintained in a prospective database. All adult patients (age $\geq 18$ years) in the database were included in the study. Only patients undergoing initial surgery were included. Redo operations were not included in the current study. Two surgeons performed all of the operations included in this study. The medical records were then reviewed for each of these patients, and the variables and outcomes of interest were documented.

The control group of asymptomatic patients was derived from the existing medical literature. A PubMed search was performed using the search terms "accessory anconeus" and "anconeus epitrochlearis." All results were reviewed for series that reported the prevalence of an anconeus epitrochlearis in asymptomatic individuals. Within the search results, all cited papers were also reviewed for series reporting prevalence. All papers reporting the prevalence of an anconeus epitrochlearis in asymptomatic individuals, whether by cadaveric study or MRI, were included.

\section{Operative Details}

Both surgeons performed open neurolysis of the ulnar nerve with or without transposition. The nerve was ex-

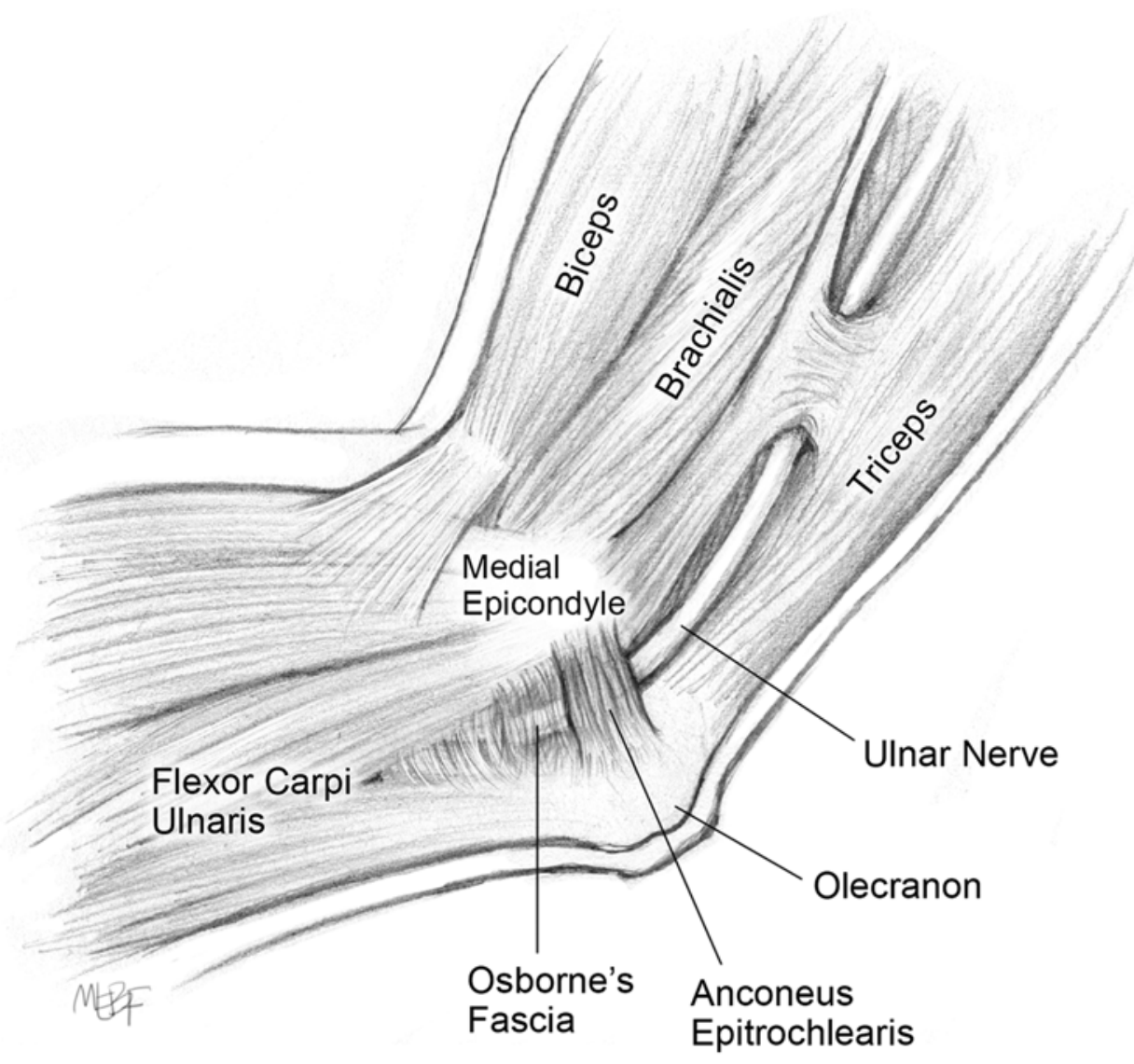

FIG. 1. Anatomical diagram showing the course of the ulnar nerve about the elbow. When present, the anconeus epitrochlearis spans from the medial epicondyle of the humerus to the olecranon process of the ulna, forming the roof of the entrance to the cubital tunnel. Copyright Thomas J. Wilson. Published with permission. 
plored and decompressed from the medial intermuscular septum through the nerve's exit from the cubital tunnel. The entrance to the cubital tunnel was visually inspected and, when present, an anconeus epitrochlearis was noted. Transposition was performed at the discretion of the surgeon with varying indications.

\section{Outcome of Interest}

The primary outcome of interest was the presence of an anconeus epitrochlearis in asymptomatic individuals versus patients with cubital tunnel syndrome. Both surgeons consistently reported in the operative report whether an anconeus epitrochlearis was encountered at the time of surgery.

\section{Variables of Interest}

The variables abstracted from the medical record included age at the time of operation, sex, operation performed, presence of an anconeus epitrochlearis, operated side, dominant hand, and whether the ulnar nerve demonstrated evidence of subluxation on examination.

\section{Statistical Analysis}

Statistical analysis was performed using commercially available software (SPSS, version 22; IBM Corp.). Quantitative data with normal distributions were expressed as the mean \pm standard deviation, and data that did not meet the normality assumption were expressed as the medians and interquartile ranges. Univariable comparisons of the continuous variables with a normal distribution were assessed using 2-sample t-tests, and continuous variables not meeting the normality assumption were assessed using the Mann-Whitney U-test. All categorical data were assessed using the chi-square test or Fisher exact test, as appropriate. A $p$ value $<0.05$ was considered statistically significant for all analyses.

\section{Results}

The demographic and surgical characteristics of the 168 patients included in the study are shown in Table 1 . The majority of patients were male. Surgery was performed with nearly equal frequency in the left versus right arm and the dominant versus nondominant arm. Subluxation of the ulnar nerve was present in $12.5 \%$ of cases. The majority of patients underwent in situ ulnar nerve neurolysis.

Among the study population who underwent ulnar nerve decompression, 9 patients were found to have an anconeus epitrochlearis at the time of surgery. One surgeon encountered an anconeus epitrochlearis in 4 of 68 operations, and the other surgeon encountered an anconeus epitrochlearis in 5 of 100 operations. Table 2 shows the demographic characteristics and surgical details for those patients who had an anconeus epitrochlearis and those who did not have this anatomical variant. There were no differences in age, sex, or operated side. Subluxation of the ulnar nerve was present in 0 of the 9 patients with an anconeus epitrochlearis versus 21 of the 159 patients (13.2\%) without an anconeus epitrochlearis, although this did not reach statistical significance. There was a trend toward
TABLE 1. Demographic details and surgical characteristics of the operations included in this study*

\begin{tabular}{lc}
\hline \multicolumn{1}{c}{ Characteristic } & Value \\
\hline No. of patients & 168 \\
\hline Mean age \pm SD, yrs & $49.1 \pm 15.4$ \\
\hline Sex & $114(67.9)$ \\
\hline Male & $54(32.1)$ \\
\hline Female & \\
\hline Side of operation & $81(48.2)$ \\
\hline Right & $87(51.8)$ \\
\hline Left & $89(53.0)$ \\
\hline Dominant arm & $21(12.5)$ \\
\hline Subluxation of ulnar nerve present & \\
\hline Operation performed & $119(70.8)$ \\
\hline In situ neurolysis & $49(29.2)$ \\
\hline Neurolysis \& transposition & $9(5.4)$ \\
\hline Anconeus epitrochlearis present & \\
\hline * Values are shown as number of patients $(\%)$ unless otherwise indicated
\end{tabular}

* Values are shown as number of patients (\%) unless otherwise indicated.

patients with an anconeus epitrochlearis undergoing neurolysis only. Finally, patients with an anconeus epitrochlearis were more likely to undergo surgery on the dominant arm versus those without an anconeus epitrochlearis. Of the patients who underwent an operation for cubital tunnel syndrome and had an anconeus epitrochlearis, 8 of 9 patients had the dominant arm operated on.

A literature review revealed 7 published studies that evaluated the prevalence of an anconeus epitrochlearis in asymptomatic individuals: 6 by cadaveric study and 1 by MRI of asymptomatic volunteers., ${ }^{2,3,5,8-10,16}$ Table 3 summarizes the included studies. In total, 634 asymptomatic patients were evaluated for the presence of an anconeus

TABLE 2. Demographic details and surgical characteristics of the operations performed on patients with and without an anconeus epitrochlearis present*

\begin{tabular}{lccc}
\hline \multicolumn{1}{c}{ Characteristic } & $\begin{array}{c}\text { Anconeus } \\
\text { Epitrochlearis } \\
(\mathrm{n}=9)\end{array}$ & $\begin{array}{c}\text { No Anconeus } \\
\text { Epitrochlearis } \\
(\mathrm{n}=159)\end{array}$ & $\begin{array}{c}\mathrm{p} \\
\text { Value }\end{array}$ \\
\hline Mean age \pm SD, yrs & $51.5 \pm 15.6$ & $49.0 \pm 15.4$ & 0.771 \\
\hline Sex & & & 0.471 \\
\hline Male & $5(55.6)$ & $109(68.6)$ & \\
\hline Female & $4(44.4)$ & $50(31.4)$ & \\
\hline Side of operation & & & 0.316 \\
\hline Right & $6(66.7)$ & $75(47.2)$ & \\
\hline Left & $3(33.3)$ & $84(52.8)$ & \\
\hline Dominant arm & $8(88.9)$ & $81(50.9)$ & 0.037 \\
\hline Subluxation of ulnar nerve & $0(0.0)$ & $21(13.2)$ & 0.604 \\
\hline Operation performed & & & 0.060 \\
\hline In situ neurolysis & $9(100.0)$ & $110(69.2)$ & \\
\hline Neurolysis \& transposition & $0(0.0)$ & $49(30.8)$ & \\
\hline
\end{tabular}

* Values are shown as number of patients (\%) unless otherwise indicated. 
epitrochlearis, and 98 of these patients had an anconeus epitrochlearis present.

The presence of an anconeus epitrochlearis occurred significantly more frequently $(\mathrm{p}<0.001)$ in the asymptomatic population (15.5\%) than in the population of patients who underwent operative intervention for cubital tunnel syndrome (5.4\%).

\section{Discussion}

Entrapment neuropathies typically occur as nerves pass beneath rigid ligamentous structures. For example, median neuropathy most commonly occurs as the median nerve passes beneath the flexor retinaculum and enters the carpal tunnel. For the lateral femoral cutaneous nerve in meralgia paresthetica, the nerve becomes entrapped as it passes beneath the inguinal ligament. For the ulnar nerve, compression often occurs as the nerve passes beneath Osborne's ligament and enters the cubital tunnel. Ulnar nerve compression can also occur at the wrist as the nerve passes beneath the palmar carpal ligament to enter Guyon's canal. A much rarer scenario involves compression of a nerve by a hypertrophied muscle secondary to overuse. An example of this form of compression neuropathy is median neuropathy secondary to compression by the 2 heads of the pronator teres as the nerve passes between them.

An anatomical variant about the elbow is the presence of an anconeus epitrochlearis. This muscle spans from the medial epicondyle to the olecranon process and replaces Osborne's ligament. The presence of an anconeus epitrochlearis has previously been suggested as a risk factor for cubital tunnel syndrome. We hypothesized, however, that by replacing a rigid ligamentous band (Osborne's ligament) with a more forgiving muscular structure (an anconeus epitrochlearis) the presence of an anconeus epitrochlearis may actually protect against the development of cubital tunnel syndrome. Furthermore, we hypothesized that in instances of compression of the ulnar nerve from an anconeus epitrochlearis, the source is likely to be hypertrophied muscle and thus is more likely to occur in the dominant arm.

We retrospectively reviewed 168 consecutive ulnar nerve decompressions. We found the prevalence of an anconeus epitrochlearis to be $5.4 \%$ among patients who underwent operative decompression. Our findings are in line with previous reports from Gervasio et al. and St. John, who reported prevalences of anconeus epitrochlearis of $3.2 \%$ (5 of 156 patients) and 1.9\% (4 of 215 patients), respectively, among patients who underwent ulnar nerve decompression. ${ }^{7,18}$ In a cohort of historical controls that consisted of asymptomatic individuals, the prevalence of an anconeus epitrochlearis was $15.5 \%$. One would expect that if an anconeus epitrochlearis is a risk factor for cubital tunnel syndrome, it would be present more frequently in patients with cubital tunnel syndrome: if it is a neutral factor, it would occur with similar frequency; if it is a protective factor, it would occur less frequently in patients with cubital tunnel syndrome than in asymptomatic individuals. We found that an anconeus epitrochlearis was present significantly less often in patients with cubital tunnel syndrome than in asymptomatic controls. We hypothesize that the mechanism of protection may be that this muscle decreases the rigidity of the entrance into the cubital tunnel.

In patients with an anconeus epitrochlearis, we found that $88.9 \%$ developed cubital tunnel syndrome in their dominant arm, while cubital tunnel syndrome occurred in the dominant arm $50.9 \%$ of the time in patients who did not have an anconeus epitrochlearis. Cubital tunnel syndrome occurred significantly more frequently in the dominant arm in patients with an anconeus epitrochlearis in comparison with those without this anatomical variant. We believe that when an anconeus epitrochlearis is the source of compression of the ulnar nerve then it likely occurs by direct compression from a hypertrophied muscle. Hypertrophy of the anconeus epitrochlearis is more likely to occur with repetitive use, which often occurs in the dominant arm. Further data are needed to fully support this hypothesis. Morgenstein et al. previously reported 4 cases of ulnar neuropathy secondary to a hypertrophied anconeus epitrochlearis resulting from occupations that required repetitive forceful extension of the elbow or prolonged periods of elbow flexion..$^{13} \mathrm{Li}$ et al. previously reported a series of 3 baseball pitchers who developed medial elbow pain and cubital tunnel syndrome secondary to hypertrophied anconeus epitrochlearis muscles. ${ }^{12}$ These reports support our hypothesized mechanism of anconeus epitrochlearis-induced ulnar neuropathy.

The anconeus epitrochlearis is thought by some to be an abnormal extension of the medial portion of the triceps muscle, but contrary to the triceps muscle it is always in-

TABLE 3. Summary of studies reporting the prevalence of an anconeus epitrochlearis in asymptomatic individuals

\begin{tabular}{lcccc}
\hline \multicolumn{1}{c}{ Authors \& Year } & $\begin{array}{c}\text { No. of Patients w/ Anconeus } \\
\text { Epitrochlearis Present }\end{array}$ & $\begin{array}{c}\text { Total } \\
\text { Specimens }\end{array}$ & $\begin{array}{c}\text { \% w/ Anconeus } \\
\text { Epitrochlearis Present }\end{array}$ & Study Method \\
\hline Bando, 1956 & 45 & 157 & 28.7 & Cadaver \\
\hline Campbell et al., 1991 & 14 & 130 & 10.8 & Cadaver \\
\hline Clemens, 1957 & 1 & 100 & 1.0 & Cadaver \\
\hline Dellon, 1986 & 11 & 104 & 10.6 & Cadaver \\
\hline Husarik et al., 2009 & 14 & 60 & 23.3 & MRI \\
\hline Mumenthaler, 1958 & 10 & 56 & 17.9 & Cadaver \\
\hline O'Driscoll et al., 1991 & 3 & 27 & 11.1 & Cadaver \\
\hline Total & 98 & 634 & 15.5 & \\
\hline
\end{tabular}


nervated by the ulnar nerve. ${ }^{8}$ The anconeus epitrochlearis, when present, forms the roof of the cubital tunnel, replacing Osborne's ligament. In fact, Testut has suggested that Osborne's ligament is a fibrous remnant of the anconeus epitrochlearis that remains after the muscle regresses. ${ }^{8}$ In cases where a hypertrophied anconeus epitrochlearis is found to be the source of compression, complete excision of the muscle and any coexisting prominent portion of the medial head of the triceps has been shown to improve the symptoms of ulnar nerve compression. ${ }^{7,15}$

Subluxation of the ulnar nerve may predispose one to the development of cubital tunnel syndrome by exacerbating the movement of the ulnar nerve relative to the fibrous intermuscular septum and Osborne's ligament that constrain the ulnar nerve, though this phenomenon has not been clearly demonstrated. Dellon previously reported that in 18 cadavers with an anconeus epitrochlearis present, none had ulnar nerve subluxation. ${ }^{5} \mathrm{He}$ further found that the presence of an anconeus epitrochlearis was associated with the medial head of the triceps covering the ulnar nerve. He used these findings to suggest that the presence of an anconeus epitrochlearis may protect the ulnar nerve from subluxation. In the present study, we found that none of the patients with an anconeus epitrochlearis had ipsilateral ulnar nerve subluxation. In comparison, $13.2 \%$ of patients without an anconeus epitrochlearis had subluxation of the ulnar nerve. This difference did not reach statistical significance, but with further data we believe that this trend may be borne out. If subluxation of the ulnar nerve does in fact predispose one to developing cubital tunnel syndrome, and the anconeus epitrochlearis prevents subluxation, this may be another mechanism by which the presence of an anconeus epitrochlearis is protective against cubital tunnel syndrome.

We have observed anecdotally that some surgeons use the presence of an anconeus epitrochlearis as an indication for ulnar nerve decompression, or at least when the diagnosis is in question the presence of an anconeus epitrochlearis will tip them in favor of surgical decompression of the ulnar nerve. However, our data suggest that the simple presence of an anconeus epitrochlearis should not be used as a surgical indication. In fact, an anconeus epitrochlearis may be protective against ulnar neuropathy. In specific instances, the anconeus epitrochlearis may be a point of compression of the ulnar nerve. However, in most cases, and in the absence of convincing ulnar neuropathy, the ulnar nerve should not be explored simply because an anconeus epitrochlearis is present.

Biomechanical data are lacking to support the hypotheses put forth in this study. Biomechanical studies that examine intraneural pressure within the ulnar nerve when an anconeus epitrochlearis is present versus absent, both statically and throughout dynamic range of motion, may help support or refute the hypothesis that an anconeus epitrochlearis reduces pressure on the ulnar nerve as it enters the cubital tunnel. Furthermore, biomechanical studies examining the excursion of the ulnar nerve throughout the range of motion when an anconeus epitrochlearis is present versus absent will help support or refute the hypothesis that an anconeus epitrochlearis reduces the risk of ulnar nerve subluxation. Larger series of patients with ulnar neuropathy secondary to a hypertrophied anconeus epitrochlearis are needed to determine the relationship of this compressive lesion to the dominant arm and activities that require repetitive forceful elbow extension or prolonged elbow flexion.

One particular weakness of the current study is the heterogeneous nature of the historical control population. The group is heterogeneous in a number of ways. First, there is wide variance in the reported prevalence of an anconeus epitrochlearis, suggesting a heterogeneous population or inconsistencies between groups in what was considered an anconeus epitrochlearis. In addition, there is heterogeneity in the method used: cadaveric studies versus MRI. In cadaveric studies, one cannot be sure that those included were truly asymptomatic without any degree of ulnar neuropathy. So, while we are considering them as asymptomatic controls, this cannot be guaranteed. Because of the limitations of the control group, the group is not directly comparable to our experimental group, thus making the findings of this study preliminary ones that require further exploration and support. In the future, more direct comparisons, such as those obtained by performing MRI in consecutive symptomatic patients and asymptomatic volunteers, may help support or refute these findings. Furthermore, such a study may also allow us to determine if symptomatic patients with an anconeus epitrochlearis do in fact have a hypertrophied muscle in the dominant arm.

\section{Conclusions}

While further studies are certainly needed, and the data presented here are preliminary, we believe our data support the notion that the anconeus epitrochlearis is a protective factor against developing cubital tunnel syndrome and support the concept that when an anconeus epitrochlearis is the source of cubital tunnel syndrome it is likely secondary to hypertrophy of the muscle from repetitive use. We believe these findings should be used as preliminary data to support further endeavors that will help support or refute our hypotheses put forth in this study.

\section{References}

1. Bando K: [Musculus epitrochleo-anconeus.] Hirosaki Igaku 7:192, 1956 (Jpn)

2. Campbell WW, Pridgeon RM, Riaz G, Astruc J, Sahni KS: Variations in anatomy of the ulnar nerve at the cubital tunnel: pitfalls in the diagnosis of ulnar neuropathy at the elbow. Muscle Nerve 14:733-738, 1991

3. Clemens HJ: Zur morphologie des ligamentum epitrochleoanconeum. Anat Anz 104:343-344, 1957

4. Cutts S: Cubital tunnel syndrome. Postgrad Med J 83:2831, 2007

5. Dellon AL: Musculotendinous variations about the medial humeral epicondyle. J Hand Surg [Br] 11:175-181, 1986

6. Descatha A, Leclerc A, Chastang JF, Roquelaure Y: Incidence of ulnar nerve entrapment at the elbow in repetitive work. Scand J Work Environ Health 30:234-240, 2004

7. Gervasio O, Zaccone C: Surgical approach to ulnar nerve compression at the elbow caused by the epitrochleoanconeus muscle and a prominent medial head of the triceps. Neurosurgery 62 (3 Suppl 1):186-193, 2008

8. Gessini L, Jandolo B, Pietrangeli A, Occhipinti E: Ulnar 
nerve entrapment at the elbow by persistent epitrochleoanconeus muscle. Case report. J Neurosurg 55:830-831, 1981

9. Hirasawa Y, Sawamura H, Sakakida K: Entrapment neuropathy due to bilateral epitrochleoanconeus muscles: a case report. J Hand Surg Am 4:181-184, 1979

10. Husarik DB, Saupe N, Pfirrmann CW, Jost B, Hodler J, Zanetti M: Elbow nerves: MR findings in 60 asymptomatic subjects-normal anatomy, variants, and pitfalls. Radiology 252:148-156, 2009

11. Kanazawa S, Fujioka H, Kanatani T, Tsunoda M, Saura R, Mizuno K: The relation between cubital tunnel syndrome and the elbow alignment. Kobe J Med Sci 40:155-163, 1994

12. Li X, Dines JS, Gorman M, Limpisvasti O, Gambardella R, Yocum L: Anconeus epitrochlearis as a source of medial elbow pain in baseball pitchers. Orthopedics 35:e1129-e1132, 2012

13. Morgenstein A, Lourie G, Miller B: Anconeus epitrochlearis muscle causing dynamic cubital tunnel syndrome: a case series. J Hand Surg Eur Vol [Epub ahead of print], 2014

14. Mumenthaler M: Die luxation des nervus ulnaris am ellenbogen. Dtsch Z Nervenheilkd 178:163-198, 1958

15. Nellans K, Tang P: Evaluation and treatment of failed ulnar nerve release at the elbow. Orthop Clin North Am 43:487494, 2012

16. O'Driscoll SW, Horii E, Carmichael SW, Morrey BF: The cubital tunnel and ulnar neuropathy. J Bone Joint Surg Br 73:613-617, 1991

17. Shi Q, MacDermid JC, Santaguida PL, Kyu HH: Predictors of surgical outcomes following anterior transposition of ulnar nerve for cubital tunnel syndrome: a systematic review. J Hand Surg Am 36:1996-2001.e1, 6, 2011

18. St. John JN: Ulnar entrapment neuropathy associated with the anconeus epitrochlearis muscle. Can J Neurol Sci 12:203-204, 1985

19. Vanderpool DW, Chalmers J, Lamb DW, Whiston TB: Peripheral compression lesions of the ulnar nerve. J Bone Joint Surg Br 50:792-803, 1968

\section{Disclosures}

The authors have no conflicts of interest to report pertaining to the materials or methods used in this study or the findings specified in this paper.

\section{Author Contributions}

Conception and design: all authors. Acquisition of data: Wilson. Analysis and interpretation of data: all authors. Drafting the article: Wilson. Critically revising the article: all authors. Reviewed submitted version of manuscript: all authors. Approved the final version of the manuscript on behalf of all authors: Wilson. Statistical analysis: Wilson. Administrative/technical/material support: Wilson. Study supervision: Wilson, Yang.

\section{Correspondence}

Thomas J. Wilson, Department of Neurosurgery, University of Michigan, 1500 E. Medical Center Dr., Rm. 3552 TC, Ann Arbor, MI 48109-5338. email: thowil@med.umich.edu. 